\title{
Ist das schweizerische Gesundheitssystem innovationsfreundlich?
}

\author{
Die Antwort auf die im Titel gestellte Frage lautet - um wieder einmal Radio Eriwan \\ die Ehre zu erweisen - «Im Prinzip Ja - aber es könnte noch besser werden».
}

Heinz Locher

Dr. rer. pol., Berater im Gesundheitswesen, Bern

Korrespondenz:

Dr. rer. pol. Heinz Locher Postfach 266 CH-3000 Bern 15

heinz@locher-bern.ch

www.locher-bern.ch

Dipl.-Kffr. Roswitha Scheidweiler RS Medical Consult GmbH Frauenberg 1

D-97980 Bad Mergentheim Tel. +49793152612

Fax +497931561226

info@rsmedicalconsult.com

www.rsmedicalconsult.com
Unser Krankenversicherungssystem ist dadurch charakterisiert, dass alle Einwohnerinnen und Einwohner unseres Landes unabhängig von ihrer wirtschaftlichen Leistungsfähigkeit Zugang zu einem ausreichenden Leistungspaket haben, für welches Tarifschutz besteht. Interessanterweise ist dieses Leistungspaket rische Krankenpflegeversicherung (mit der Möglichkeit zu Einschränkungen und Auflagen) [2] erleichtert den raschen Zugang zu deren Finanzierung durch die obligatorische Krankenpflegeversicherung. Dieser Umstand rechtfertigt das «Ja» in der Antwort zur im Titel gestellten Frage.

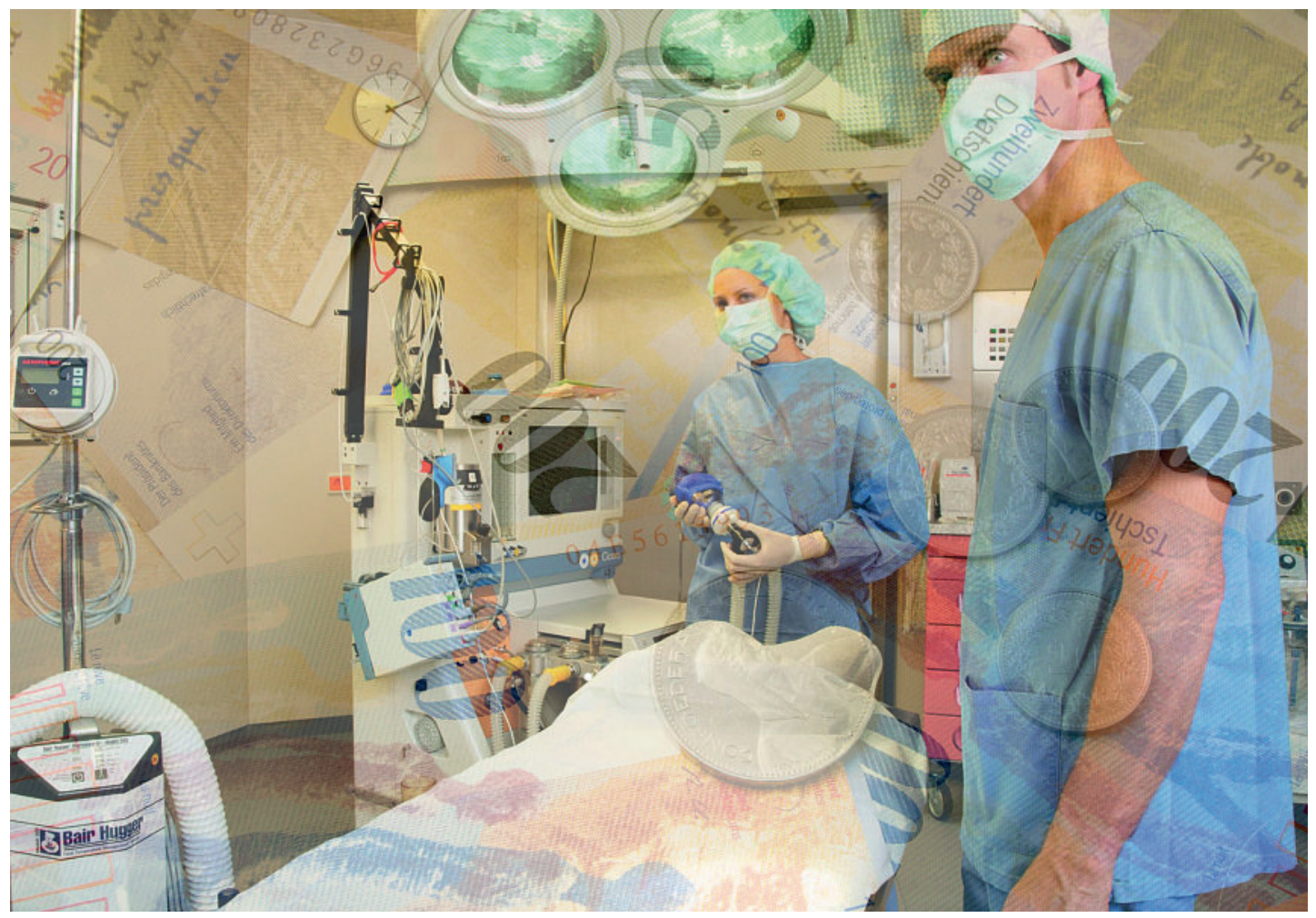

nur in Teilbereichen umschrieben, so etwa durch die Spezialitätenliste bei Medikamenten. Für ärztliche Leistungen besteht keine Positivliste, es gilt das sogenannte Vertrauensprinzip.

\section{Nicht die beste aller (Krankenversicherungs-) Welten}

Der Anhang zur Krankenpflege-Leistungsverordnung (KLV) [1] konkretisiert dieses in Einzelfällen, es handelt sich dabei um eine eklektische Mischung zwischen einer Positiv- und einer Negativliste. Das faktische Fehlen einer Positivliste für die Zulassung von ärztlichen Leistungen zur Finanzierung durch die obligato-

\section{Verbesserungspotential nur sehr beschränkt ausgeschöpft}

Trotzdem leben wir nicht in der besten aller (Krankenversicherungs-)Welten. In einem Bericht der Parlamentarischen Verwaltungskontrolle vom 21. August 2008 zum Thema «Bestimmung und Überprüfung ärztlicher Leistungen in der obligatorischen Krankenpflegeversicherung» [3] wird festgehalten, dass «das Vertrauensprinzip aber nur gerechtfertigt (ist), wenn Leistungen, die hinsichtlich der WZW-Kriterien umstritten sind, systematisch evaluiert und im negativen Fall aus der OKP ausgeschlossen werden. Diese Ausfilterung funktioniert in der Praxis - trotz Verbesse- 
rungen in jüngster Zeit - nicht hinreichend.» Dieses erhebliche Verbesserungspotential ist auch heute noch nur in sehr beschränktem Masse ausgeschöpft, was das «aber es könnte noch besser werden» in der Antwort zur im Titel gestellten Frage rechtfertigt.

$\mathrm{Zu}$ zusätzlicher Aktualität ist das Thema im Zusammenhang mit dem SwissDRG-System gekommen, das den medizinischen Fortschritt nicht behindern soll. Durch geeignete prozedurale Vorkehrungen ist dieses wichtige Anliegen problemlos umsetzbar: Bezeichnung der antragsberechtigten Organisationen, klare Verfahrensregeln, rasche Entscheide, provisorische Zulassung mit Auflagen (z. B. Begleitforschung, Registerpflicht). Es bleibt zu hoffen, dass die entsprechenden Regelungen nun rasch getroffen und umgesetzt werden.

1 Krankenpflege-Leistungsverordnung (KLV), Anhang, Amtliche Sammlung 832.112.31.

2 Verordnung Krankenversicherung, (KVV), Art. 33, Amtliche Sammlung 832.102.

3 Bestimmung und Überprüfung ärztlicher Leistungen in der obligatorischen Krankenpflegeversicherung. Bericht der Parlamentarischen Verwaltungskontrolle zuhanden der Geschäftsprüfungskommission des Nationalrates, Bundesblatt Nr. 30, 28. Juli 2009, S. 5589.
Symposium Zukunft der medizinischen Versorgung und der kantonalen Gesundheitspolitik

Der «Countdown» läuft: Wenige Monate trennen uns nur noch vom «Tag X», dem 1. Januar 2012, an dem weitere wichtige Teile der revidierten Gesetzgebung zur Spitalfinanzierung in Kraft treten, allem voran das Patientenklassifikations- und Leistungsfinanzierungssystem SwissDRG. Noch sind verschiedene Fragen offen oder bedürfen doch ihrer Konkretisierung, so die Zulassung und Finanzierung medizinischer Innovationen, die Finanzierung von Investitionen sowie der Weiterbildung in den Medizinalberufen. Auch wenn diese und weitere aktuelle Fragen das Tagesgeschehen prägen und viele Kräfte binden, sollten ihre Einordnung und ihre Auswirkungen auf Grundsatzfragen nicht vernachlässigt werden. Der «Charme des eklektischen Pragmatismus», der unseren (gesundheits-)politischen Alltag charakterisiert, birgt die Gefahr einer ordnungspolitischen Verwahrlosung. Eine Rückbesinnung auf die ordnungspolitischen Grundlagen unseres Gesundheitssystems ist deshalb dringender denn je.

Unter den Referenten des Zürcher Symposiums finden sich auch zwei Schweizer Experten mit langjähriger Erfahrung in Deutschland: Josef Rohrer, Geschäftsführer eines deutschen Grossklinikums, und Prof. Dr. med. Marc Reymond, Viszeralchirurg und Chefarzt am Evangelischen Krankenhaus in Bielefeld. Der Anlass wird moderiert von Prof. Dr. oec. Tilman Slembeck, Professor für Volkswirtschaftslehre, Zürcher Hochschule für Angewandte Wissenschaften und Universität St. Gallen, sowie Frau Roswitha Scheidweiler, Dipl.Kffr., RS Medical Consult GmbH.

Die Veranstaltung wird in Kooperation mit der Konferenz der Ostschweizer Ärztegesellschaften organisiert.
Weitere Informationen zum Symposium und OnlineAnmeldung unter www.rsmedicalconsult.com

\footnotetext{
Programmübersicht des Symposiums vom 23. Juni 2011 im Kongresshaus Zürich

9.00 Uhr Begrüssung

9.05 Uhr Dr. iur. Thomas Heiniger: Grusswort / offizielle Eröffnung

09.20 Uhr Dr. rer. pol. Heinz Locher: Finanzierung von Innovationen im schweizerischen Gesundheitssystem (Einführungsreferat)

09.40 Uhr Dr. iur. Carlo Conti: Stand der Einführung von SwissDRG - was gibt es noch zu tun?

10.00 Uhr Joseph Rohrer: Besteht eine Notwendigkeit zur Änderung der Managementinhalte ab 2012?

10.20 Uhr Karl Heinz Tuschen: Erfahrungen aus der DRG Einführung in Deutschland

10.40 Uhr Prof. Dr. med. Prof. h.c. Marc A. Reymond: Medizinische Innovationen und ärztliche Weiterbildung unter DRG-Bedingungen

11.15 Uhr Plenumsdiskussion mit den Referenten und den Podiumsgästen Andreas Faller, lic. iur. Advokat, Dr. phil. nat. Bernhard Wegmüller und Stefan Kaufmann

12.45 Uhr Gemeinsames Mittagessen

13.45 Uhr Dr. rer. pol. Heinz Locher: Ordnungspolitik im schweizerischen Gesundheitswesen (Einführungsreferat)

14.00 Uhr Prof. Dr. Herbert Rebscher: Wettbewerb im deutschen Gesundheitswesen - wie sieht die Praxis aus?

14.20 Uhr Dr. med. Daniel Germann: Zwischen Wettbewerb und öffentlichem Versorgungsauftrag

14.35 Uhr Dr. med. Peter Wiedersheim: Wettbewerb aus Sicht der praktizierenden Ärzteschaft

14.50 Uhr Prof. Dr. Robert Leu: Erfahrungen aus dem Ausland - was kann die Schweiz daraus lernen?

15.05 Uhr Thomas B. Cueni: Wettbewerb im dezentralisierten Gesundheitswesen

15.20 Uhr Dr. rer. pol. Melchior Buchs: Herausforderungen für die Medtechbranche unter DRG ab 2012

15.35 Uhr Prof. Dr. Thomas D. Szucs: Regulierung des Wettbewerbs aus Sicht der Helsana Krankenversicherung

15.50 Uhr Diskussions- und Fragerunde mit den Referenten des Nachmittages und Dr. med. Urs Stoffel als Podiumsgast

17.20 Uhr Schlusswort; anschliessend Gedankenaustausch unter den Teilnehmenden beim Schlussaperitif

Der Schweizerische Ärzteverlag EMH ist Medienpartner des Symposiums.
} 